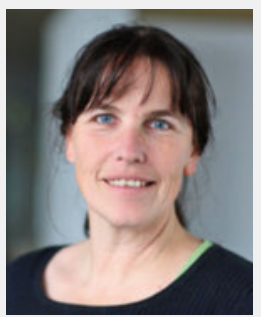

Beate Schweizer, Stuttgart

\section{Neues Jahr, neues Layout, neuer Index}

Liebe Leserinnen und Leser,

Ende 2016 erhielt der Georg Thieme Verlag erfreuliche Post von Thomson Reuters: Sie haben die Zeitschrift Flugmedizin Tropenmedizin Reisemedizin in ihren Index „Emerging Sources Citation Index, ESCl“ aufgenommen.

In den ESCl werden laut Thomson Reuters „qualitativ hochwertige, peer-reviewte Publikationen von regionaler Bedeutung beziehungsweise aus sich entwickelnden Fachgebieten nach einer initialen Begutachtung aufgenommen. Grundlage für diese Entscheidung sind Publikationsstandards, Inhalte sowie Zitationshäufigkeiten der Zeitschrift." Mit dieser Listung hat die FTR zwar noch keinen Impact Factor, wird aber für eine Aufnahme in den Science Citation Index in Betracht gezogen, aus dem die Berechnung des Journal Impact Factors erfolgt. Dafür wird die FTR nun, nach der initialen positiven Begutachtung, weiter beobachtet.

Ich freue mich sehr über diese Anerkennung. Ermöglicht wurde die Qualität der bisherigen Inhalte durch das große Engagement der Gesellschaften, ihren Vertretern im Editorial Board und allen Autoren. Es ist außerdem ein Ansporn, Ihnen weiterhin gemeinsam die Inhalte und Informationen anzubieten, die für Sie wichtig sind.

Das Jahr 2017 beginnt mit einer weiteren Neuheit, die Sie beim Lesen dieses Editorials bereits vor sich haben: Ausgabe 1 startet - wie viele weitere Thieme Zeitschriften mit einem neuen Layout in das neue Jahr. Eine aktuelle Marktforschung des Verlags und viele Gespräche mit Lesern haben geänderte Lesegewohnheiten gezeigt: Aufgeräumte, klare Strukturen, um die Fachinhalte bestmöglich aufnehmen zu können, sind neben einer relevanten Themenauswahl demnach besonders wichtig. Auf diese Ansprüche konzentriert sich das neue Farbkonzept und die neuen Schriften, die nicht nur für gedruckte sondern auch für elektronische Medien geeignet sind.

Die Autoren dieser Ausgabe haben das neue Layout bereits vor Ihnen kennengelernt, als wir die Druckfassungen erstellt haben. Ich danke allen Autoren für ihre Bereitschaft, ihre gesetzten Beiträge in teilweise sehr kurzen Zeiträumen fertigzustellen.

In dieser Ausgabe sind unter anderem 3 Kasuistiken veröffentlicht. Die Themen sind „Unfall mit Thoraxverletzung nach Sturz ins Wasser“, „Windpocken an Bord eines Kauffahrteischiffs“ sowie „Assistancemedizin: Betreuung nach Arbeitsunfall in Polen“. Außerdem informiert Martin Schwiersch darüber, welche Maßnahmen es Menschen bei Bergtouren ermöglichen, in kritischen Situationen vernünftige Entscheidungen zu treffen. Ein weiteres Thema ist das Pellagra, das aktuell eine Bedrohung für die wachsende Anzahl von Flüchtlingen weltweit ist. Andreas Montag erläutert Hintergründe und Therapiemöglichkeiten der Erkrankung, die durch Mangel- und Fehlernährung hervorgerufen wird.

Ich wünsche Ihnen allen eine interessante Lektüre. 